\title{
Priorities for disclosure of the elements of informed consent for research: a comparison between parents and investigators
}

\author{
ALAN R. TAIT PhD, TERRI VOEPEL-LEWIS MSN, RN, \\ ANGELA ROBINSON BS AND SHOBHA MALVIYA MD \\ Department of Anesthesiology, University of Michigan Health Systems, Ann Arbor, MI, USA
}

\section{Summary}

Background: Disclosure is a key element of the informed consent process. This study examines and compares the priorities for disclosure of the elements of informed consent between parents of paediatric research subjects and investigators.

Methods: The study sample comprised 184 parents who had been approached for permission to allow their child to participate in a clinical anaesthesia or surgery study. Parents were asked to rank 11 elements of informed consent that they believed were most important for them to know before allowing their child to participate in a research study. In addition, 38 investigators were asked to rank, in order of importance, the same elements that they felt were most important for parents to know.

Results: The results showed that risk was the most important element considered by both parents and investigators. However, parents placed significantly greater importance on knowledge of the potential benefits to their child (direct) and to other children (indirect) compared with investigators, and less importance on the details of the protocol and the element of voluntariness.

Conclusions: These results demonstrated differences in the priorities for disclosure of the elements of consent between parents and investigators. As such, they may be important in directing the investigator to focus on the elements that are most important to parents and thus maximize their ability to provide truly informed consent.

Keywords: informed consent; paediatric research; ethics; disclosure

Correspondence to: Alan R. Tait, Department of Anesthesiology, University of Michigan Health Systems, 1500 East Medical Center Drive, Ann Arbor, MI 48109, USA (e-mail, atait@umich.edu).

\section{Introduction}

The importance of disclosure as a key component of the informed process promulgated the development of several standards of disclosure, including the 
reasonable doctor standard', the 'hypothetical reasonable person standard' and the 'subjective standard' (1). As such, all investigators have a duty to disclose material information regarding studies involving human subjects. Each disclosure requires the incorporation of several key informational elements, including the risk and benefits, the purpose of the study and a description of what is to be carried out. Failure to provide complete disclosure such that the subject cannot make an informed decision is tantamount to negligence (2).

Although the decision of a subject or their surrogate to decline participation in a research study must be respected, nonresponse or nonparticipation may introduce a significant selection bias (3). It is therefore important to optimize the quality of information provided to research subjects such that the ethical and scientific needs of the study are met. This study was designed to identify the elements of consent that parents consider most important for them to understand prior to making decisions regarding their child's participation in a research study, and to examine if they differ from the elements considered most important by research personnel. An understanding of these differences may help researchers focus on the elements of disclosure considered most important by parents and hence maximize their ability to make an informed decision.

\section{Methods}

The study was approved by the University of Michigan's Institutional Review Board. The study population comprised two groups. Group 1 included consecutive parents/guardians who had been approached for permission to allow their child to participate in any one of a number of anaesthesia or surgery research studies presently in progress at our institution. Information regarding each study was presented verbally in addition to the written consent document. The majority of parents were approached on the day of their child's surgery. Regardless of whether or not the parents had consented to their child's participation in one of the studies, they were invited to participate in this study which required them simply to rank, in order of importance, 11 elements of consent that they believed they needed to know before deciding to allow their child to participate in a research study. These 11 elements are those typically contained within informed consent documents and included: (i) study purpose; (ii) protocol (what will be done); (iii) freedom to withdraw; (iv) risks; (v) benefits to their child (direct benefits); (vi) benefits to other children (indirect benefits); (vii) alternative treatments or procedures; (viii) voluntariness; (ix) duration of participation; (x) whom to contact with questions/problems; and (xi) confidentiality.

Subjects were asked to rank each element where $1=$ most important and $11=$ the least important . The order in which these elements were presented was changed during the study to avoid any patterned responses. Demographic information, including age (parent and child), gender, race/ethnic origin, level of education and income, were also collected.

Group 2 comprised research personnel, including principal investigators, research assistants and research nurses who were actively involved in recruiting paediatric subjects and obtaining informed consent for anaesthesia and surgery studies. These individuals were identified from departmental anaesthesia and surgery personnel directories. Consistent with group 1, the research personnel were asked to rank the same 11 elements in the order that they believed was most important for parents to know prior to making a decision regarding their child's participation in research. Demographic information, including title, degree, years in clinical research, gender and race/ethnic origin, was also collected.

\section{Statistical analysis}

Continous data were analysed using unpaired $t$-tests. Comparison of the rankings of importance between groups was analysed by the Mann-Whitney $U$-test. Categorical data were analysed by chisquare with Fisher's exact test as appropriate. $P<0.05$ was considered statistically significant.

\section{Results}

Rankings were obtained from 184 parents; 147 from parents who had consented to their child's participation in one of the anaesthesia or surgery studies (consenters) and 37 from those who declined (nonconsenters). In addition, rankings were obtained from 38 research personnel. The demographics of 


\begin{tabular}{|c|c|c|c|}
\hline & $\begin{array}{c}\text { Consenters } \\
(\mathrm{n}=143)\end{array}$ & $\begin{array}{l}\text { Nonconsenters } \\
\quad(\mathrm{n}=35)\end{array}$ & $\begin{array}{c}\text { All parents } \\
(\mathrm{n}=184)\end{array}$ \\
\hline Parent's age (year) & $35.7 \pm 7.6$ & $34.6 \pm 6.6$ & $35.5 \pm 7.4$ \\
\hline Child's age (year) & $6.0 \pm 4.6$ & $4.7 \pm 3.5$ & $5.8 \pm 4.4$ \\
\hline Child's health* & $8.3 \pm 1.8$ & $8.4 \pm 1.9$ & $8.3 \pm 1.8$ \\
\hline Child's sex (M/F\%) & $58.2 / 41.8$ & $74.3 / 25.7$ & $61.3 / 38.7$ \\
\hline \multicolumn{4}{|l|}{ Race, $n(\%)$} \\
\hline Caucasian & $124(86.7)$ & $31(88.6)$ & $155(87.1)$ \\
\hline African American & $6(4.2)$ & $2(5.7)$ & $8(4.5)$ \\
\hline Hispanic & $3(2.1)$ & $0(0.0)$ & $3(1.7)$ \\
\hline Other & $9(6.3)$ & $2(5.7)$ & $11(6.2)$ \\
\hline \multicolumn{4}{|l|}{ Education level, $n(\%)$} \\
\hline$\leq$ High school graduate & $42(28.6) \dagger$ & $3(8.6)$ & $45(24.7)$ \\
\hline Some college & $34(23.1)$ & $11(31.4)$ & $45(24.7)$ \\
\hline$\geq$ College graduate & $71(48.3)$ & $21(60.0)$ & $92(50.5)$ \\
\hline \multicolumn{4}{|l|}{ Income level, $n(\%)$} \\
\hline$<\$ 0-\$ 29000$ & $31(23.1)$ & $4(11.4)$ & 35 (21.5) \\
\hline$\$ 30000-\$ 69000$ & $53(39.6)$ & $9(25.7)$ & $62(38.0)$ \\
\hline$>\$ 70000$ & $50(37.3)$ & $16(45.7)$ & $66(40.5)$ \\
\hline Prior research subject-child & $29(19.1)$ & $6(16.2)$ & $35(18.5)$ \\
\hline Prior research subject-parent & $39(25.7)$ & $9(24.3)$ & $48(25.4)$ \\
\hline
\end{tabular}

Table 1

Parent and child demographics

Data are expressed as mean \pm SD and percentage. *Based on a $0-10$ scale where $10=$ very healthy. $+P<0.05$ versus nonconsenters.

the parents and children are presented in Table 1. There were no differences between consenters and nonconsenters with respect to age, race and income level; however, nonconsenters were significantly more likely to have education beyond high school than consenters $(P=0.04)$. Table 2 describes the demographics of the research personnel.

The rankings of importance of each of the elements of consent for consenters and nonconsenters are shown in Table 3. Although both groups rated

Table 2

Demographics of the research personnel $(n=38)$

\begin{tabular}{lc}
\hline & $\mathrm{n}(\%)$ \\
\hline Research personnel & \\
Principal investigators & $21(55.3)$ \\
Research assistants & $10(26.3)$ \\
Research nurses & $3(7.9)$ \\
Undergraduate students & $3(7.9)$ \\
Research fellow & $1(2.6)$ \\
Gender (M/F\%) & $26.3 / 73.7$ \\
Race & \\
Caucasian & $27(71.1)$ \\
African American & $4(10.5)$ \\
Hispanic & $1(2.6)$ \\
Other & $6(15.8)$ \\
Years in research & \\
$<5$ & $16(42.1)$ \\
$5-10$ & $15(39.4)$ \\
$11-15$ & $6(15.8)$ \\
$16-20$ & $1(2.6)$ \\
\hline
\end{tabular}

risks as the most important element, consenters rated the benefits to others as significantly more important than nonconsenters. Table 4 compares the priority rankings of each element between parents and research personnel. Once again, risk was rated as the most important element by both groups. However, parents placed greater importance on knowledge of the benefits to their child and other children compared with the investigators, and less importance on the details of the protocol and the issue of voluntariness. There were no statistical differences in rankings among the research person-

Table 3

Priority rankings between consenters and nonconsenters

\begin{tabular}{lll}
\hline Elements of consent & Consenters & Nonconsenters \\
\hline Risks & $2.4 \pm 2.4(1)$ & $2.0 \pm 2.2(1)$ \\
Benefits to child & $3.7 \pm 2.4(3)$ & $3.0 \pm 1.7(2)$ \\
Protocol/procedures & $4.7 \pm 2.8(4)$ & $4.9 \pm 2.9(4.5)$ \\
Study purpose & $5.0 \pm 2.8(4)$ & $5.5 \pm 2.8(4.5)$ \\
Benefits to others & $5.2 \pm 2.4(5)^{*}$ & $7.2 \pm 2.2(6.5)$ \\
Duration of study & $6.6 \pm 2.2(6)$ & $6.9 \pm 2.2(7)$ \\
Voluntariness & $6.6 \pm 2.8(7)^{*}$ & $5.4 \pm 2.8(5)$ \\
Freedom to withdraw & $7.0 \pm 2.9(7)$ & $6.9 \pm 3.0(7)$ \\
Alternatives & $7.3 \pm 2.8(8)$ & $6.6 \pm 3.0(6.5)$ \\
Whom to contact & $8.3 \pm 2.2(9)$ & $8.4 \pm 2.4(9)$ \\
Confidentiality & $8.6 \pm 2.8(10)$ & $8.3 \pm 2.2(8)$ \\
\hline
\end{tabular}

Data are expressed as mean $\pm \mathrm{SD}$ (median). ${ }^{*} P<0.05$ versus nonconsenters. 
Table 4

Priority Rankings between Parents and Investigators [mean \pm SD (median)]

\begin{tabular}{lll}
\hline Elements of consent & All parents & Investigators \\
\hline Risks & $2.3 \pm 2.3(1)$ & $2.6 \pm 2.0(2)$ \\
Benefits to child & $3.5 \pm 2.3(3)^{*}$ & $6.0 \pm 2.8(6)$ \\
Protocol/procedures & $4.7 \pm 2.8(4)^{*}$ & $2.8 \pm 1.5(2)$ \\
Study purpose & $5.1 \pm 2.8(4)$ & $4.1 \pm 2.8(4)$ \\
Benefits to others & $5.7 \pm 2.5(5)^{*}$ & $8.3 \pm 2.1(9)$ \\
Duration of study & $6.6 \pm 2.2(7)$ & $7.2 \pm 2.4(8)$ \\
Voluntariness & $6.3 \pm 2.8(7)^{*}$ & $4.3 \pm 2.8(4)$ \\
Freedom to withdraw & $6.9 \pm 2.9(7)$ & $6.0 \pm 2.4(6)$ \\
Alternatives & $7.1 \pm 2.8(8)$ & $6.5 \pm 2.8(6.5)$ \\
Whom to contact & $8.4 \pm 2.3(9)$ & $8.3 \pm 2.7(9)$ \\
Confidentiality & $8.6 \pm 2.7(10)$ & $8.2 \pm 2.6(9)$ \\
\hline
\end{tabular}

${ }^{*} P<0.05$ versus investigators.

nel with respect to gender, years in research and title (faculty investigators versus staff).

Twenty-two percent of the studies presented to the parents were considered to have a therapeutic intent. Parents of children recruited into studies with therapeutic intent ranked the benefits to their child as significantly more important $[2.6 \pm 2.1$ (median 2) versus $3.7 \pm 2.2$ (median 3 ) $P=0.016$ ] and the protocol as less important $[5.5 \pm 2.9$ (median 5 ) versus $4.3 \pm 2.6$ (median 4) $P=0.014]$ compared with those involved in studies with no therapeutic intent.

\section{Discussion}

A recent review of research protocols by the National Bioethics Advisory Commission (NBAC) identified several areas of concern regarding the informed consent process, including potentially coercive recruitment practices, failure to assess decision-making capacity and the downplaying of risks (4). Many of these dubious practices may result from investigators who feel under pressure (intrinsic or extrinsic) to expedite subject recruitment. Although it is every investigator's goal to maximize recruitment rates in order to provide a representative sample of sufficient size to achieve statistical power, it must be achieved through the design of ethically sensitive protocols involving complete and honest disclosure. In order that this can be achieved, investigators must be sensitive to the elements of disclosure that are most important to the subject/ surrogate in order for them to make an informed choice.
It should be noted that participation in more than one study is not generally advocated by our Institutional Review Board; however, this caveat may be waived if the investigators agree that the risk of an additional study is no more than minimal, that the subject will not be harmed and that the outcome of the studies will not be disturbed. Furthermore, in our study, the parent was the research subject whereas, in the initial study, the child was the subject. A second issue regarding consent for this study is that despite the fact that some parents declined their child's participation in one of the studies, they chose to participate in ours. We believe that this may have reflected differences in the parents' perception of risk between the two studies. Since the risk of our study was minimal and, in our experience, parents are extremely altruistic, they may have chosen our study despite declining their child's participation.

This study suggests that there are differences between parents and investigators regarding the relative importance of certain disclosed elements. Although both parents and investigators in our study considered the risks as the most important element for disclosure, investigators felt that the disclosure of benefits (direct and indirect) was of significantly lower priority than the parents. In a study by Susman et al. eleven paediatricians ranked risks, benefits to the subject and procedures as the three most important elements, and freedom to withdraw, knowledge of participation and benefit to others as the three least important (5). These results are similar to ours and reinforce the disparity between the relative importance of risk and benefit (particularly indirect). The discrepancy between risk and benefit is important since the risk/benefit ratio is one of the principal measures used by Institutional Review Boards in their review of study protocols. The finding that disclosure related to confidentiality was deemed least important by both parents and investigators was somewhat surprising given the importance placed on this element by both Institutional Review Boards and the National Institutes of Health. However, this finding may be a function of receiving care at a large academic medical centre where patients expect some sharing of their medical information between health care providers and services and recognize their potential for becoming a research subject. 
The results also showed that the consenters ranked the benefits to others as significantly more important than nonconsenters which may suggest a greater degree of altruism among those willing to have their child participate in a study. However, there are several other factors that influence parents' decisions to allow their children to be involved in research projects, including the perceived importance of the study, the risks and benefits and whether their child had participated in a previous study (6).

Although many studies do not provide a direct benefit to the subject, there may be significant indirect benefits for subsequent patients should a therapy or intervention prove efficacious. A previous study from our institution showed that the risk/benefit was a principal factor influencing parents' decisions to allow their children to participate in a study (6). Furthermore, the study showed that the majority of parents approached for permission to allow their child to participate in a research study were genuinely altruistic, with a keen desire to help future children. As such, it may behove investigators to spend extra time to ensure that the benefits, both direct and indirect, are given as much consideration as the risks and that the parents and child fully understand their significance.

Regardless of the priority rankings of each element examined in this study, it must be noted that all elements are considered important and required for disclosure. Furthermore, it could be argued that, despite statistically significant differences in the priority ranking between groups, clinical significance is less pronounced. However, this study is the first to address this issue in a surgical population of children and provides important information regarding the relative importance of disclosure elements in the consent process. As such, it is hoped that these results will help research personnel to focus on those elements that are most important to parents in order to maximize parental understanding, and ensure that the rights of the research subject are protected.

\section{Acknowledgements}

Supported by a grant from the National Institutes of Health, NIGMS (GM 61971). The authors would like to thank Janelle Marshall, Melanie Wagner, Anna Halbeisen and Megan Harris-Linton for their help with subject recruitment and data collection.

\section{References}

1 Beauchamp TL, Childress JF. In: Beauchamp TL, Childress JF, eds. Principles of Biomedical Ethics, 4th edn. New York, NY: Oxford University Press, 1994.

2 Waisel DB, Truog RD. Informed consent. Anesthesiology 1997; 87: 968-978.

3 Goodman NW, Black AMS. Refusal rates for clinical trials. $\mathrm{Br}$ J Anaesth 1986; 58: 938-939.

4 Report and Recommendations of the National Bioethics Advisory Board. Research Involving Persons with Mental Disorders That May Affect Decisionmaking Capacity. The National Bioethics Advisory Board, 1998.

5 Susman EJ, Dorn LD, Fletcher JC. Participation in biomedical research. The consent process as viewed by children, adolescents, young adults, and physicians. J Pediatr 1992; 121: 547552.

6 Tait AR, Voepel-Lewis T, Siewert M et al. Factors that influence parents' decisions to consent to their child's participation in clinical anesthesia research. Anesth Analg 1998; 86: 50-53.

Accepted 13 November 2001 